\title{
Vitamin A Supplementation for Prevention and Treatment of Malaria during Pregnancy and Childhood: A Systematic Review and Meta-analysis
}

\author{
Mohammad Yawar Yakoob ${ }^{1, *}$, Murad Qadir ${ }^{1, \dagger}$, Omm-e-Hany $^{2}$ \\ ${ }^{1}$ Department of Community Health Sciences, Jinnah Medical and Dental College, Karachi, Pakistan \\ ${ }^{2}$ Institute of Environmental Studies, University of Karachi, Karachi, Pakistan
}

ART I C E I N F O
Article History
Received 13 December 2017
Accepted 25 August 2018
Keywords
Childhood
preventive
pregnancy
randomized controlled trials
supplementation
treatment
vitamin A

\section{ARTICLE INFO}

Received 13 December 2017

\section{Keywords}

andomized controlled trials

treatment

vitamin A

\begin{abstract}
Animal studies have shown that vitamin A plays a role in immunity and protection against infectious diseases. Its role reducing incidence of diarrhea and measles, and childhood mortality is known, but its role in relation to malaria is unclear. Thus, a comprehensive, systematic literature search was conducted on PubMed and Cochrane Library to identify randomized controlled trials (RCTs) on the role of vitamin A during pregnancy and childhood for prevention and treatment of malaria. A total of 107 titles/abstracts were identified, of which 15 articles (11 studies) were selected for final inclusion. Based on the meta-analysis, vitamin A supplementation during pregnancy had no benefit for placental infection (relative risk $=1.09 ; 95 \%$ confidence interval (CI), 0.95-1.25; fixed effects, $I^{2}=0 ; 2$ RCTs). Similarly, there was no effect on peripheral parasitemia or episodes of new clinical malaria. Preventive vitamin A supplementation in children younger than 5 years did not reduce the incidence of peripheral parasitemia or malaria mortality (latter rate ratio $=0.49 ; 95 \%$ CI, $0.07-3.26$; random effects, $I^{2}=72 \%, 2$ RCTs). Vitamin A as an adjunct treatment for cerebral or severe malaria in children did not have benefit on survival, fever resolution time, parasite clearance time, or incidence of neurological or other complications. Vitamin A has no benefit for malarial infection either as prevention or treatment in pregnancy or childhood based on RCT evidence.
\end{abstract}

() 2018 Atlantis Press International B.V This is an open access article under the CC BY-NC license (http://creativecommons.org/licenses/by-nc/4.0/)

\section{INTRODUCTION}

According to the Global Burden of Disease and World Health Organization (WHO) 2016 estimates, although the incidence of malaria has decreased by $21 \%$ since 2010, it still accounts for 212 million cases and 429,000 deaths globally, with an attributable fraction of $1.32 \%$ of total deaths [1,2]. Malaria is a major public health burden with $90 \%$ of cases occurring in the African continent, followed by Southeast Asia (7\%) and the Eastern Mediterranean region (2\%) [2]. Malarial infection is common among the poor and underprivileged populations in developing countries, mostly affecting vulnerable adults, pregnant women, and children [3,4]. This necessitates making prevention and treatment efforts a priority in combatting this disease. Because there is a link between poverty and undernutrition, it is hypothesized that nutrition may play an important role in either primary prevention or treatment of malaria. Undernutrition is accompanied by deficiency of different micronutrients, and it is not clear if supplementation of single or multiple micronutrients can be of benefit or even increase the risk of infection in the setting of adequate nutrition.

Vitamin $\mathrm{A}$ is known to modulate innate and adaptive immune responses and is vital for host immunity against different

${ }^{*}$ Corresponding author. Present address: Indus Hospital Research Center, Indus Health Network, Karachi, Pakistan.Emails:myyakoob@mail.harvard.edu; yawar.yaqoob@tih.org.pk ${ }^{\dagger}$ Present address: Department of Community Health Sciences, United Medical and Dental College, Karachi, Pakistan infectious diseases. Vitamin A supplementation in randomized controlled trials (RCTs) is known to protect against incidence of diarrhea and measles in children younger than 5 years (by $15 \%$ and $50 \%$, respectively-low to moderate quality evidence), and also reduce diarrhea-related and all-cause mortality [5]. Similarly, the effect of vitamin A supplementation on maternal clinical infections (mostly puerperal sepsis) is borderline significant with low-quality evidence [6]. It may, therefore, also play its part in malaria prevention and reduction in mortality due to malaria, the role of which is unclear. Various mechanisms have been proposed from animal studies for the protective role of vitamin A. Vitamin A may enhance phagocytosis of erythrocytes harboring the malarial parasite and attenuate the proinflammatory cytokine responses to infection [3]. Further animal model studies have also shown that vitamin A may play a major role in the functioning of the immune system, including lymphopoiesis, apoptosis, production of antibody, and the function of other immune cells such as neutrophils, natural killer cells, monocytes or macrophages, and lymphocytes [7].

The effect of vitamin A in preventing and treating malarial infections has not previously been studied in the form of a systematic review and meta-analysis based on evidence from RCTs. A meta-analysis would increase the power and allow for potentially statistically significant effects that have not been observed in individual studies. Moreover, this evidence has not been compiled in a comprehensive, organized, and simple manner to date. 
We therefore conducted this systematic review and meta-analysis to evaluate the effects of vitamin A supplementation during both pregnancy and childhood (younger than 5 years) for malaria prevention and treatment.

\section{MATERIALS AND METHODS}

A comprehensive, systematic literature search was conducted in July 2017 on PubMed and the Cochrane Library to identify RCTs on vitamin A supplementation in relation to malaria. The complete search term used was ("vitamin A", suppl*, and malaria). Including synonym terms for vitamin A (such as retinol OR carotenoids) and malaria (such as plasmodium OR "remittent fever") without suppl ${ }^{*}$ yielded 275 titles/abstracts but no relevant additional RCTs. We also searched Google Scholar for gray literature with the original search strategy (first 100 hits) but found no additional studies.

We used the following inclusion/exclusion criteria. (1) RCTs where vitamin A supplementation should be the only difference between the intervention and placebo groups. If vitamin A was given with any other supplement such as zinc $(2 \times 2$ factorial design $)$, then those two groups were included that differed only in vitamin A (if no interaction with zinc) or vitamin A versus placebo groups (if an interaction). (2) The supplementation should be during pregnancy irrespective of the trimester or month of pregnancy. RCTs on post-partum supplementation were excluded. (3) Supplementation in children younger than 5 years. For those studies where age range exceeded 5 years, we included the overall results and did not report dissociated data for children younger than 5 years because such data were not found in any of the RCTs.

The primary/secondary outcomes are as follows. (1) The primary outcomes considered for prevention of vitamin A during pregnancy were incidence of clinical malaria and peripheral parasitemia. The secondary outcome considered was histopathological infection. (2) The primary outcomes considered for prevention in children were incidence of clinical malarial episodes and malaria-specific mortality. For treatment of malaria, the primary outcomes considered were survival and time to resolution of infection (fever). Secondary outcome was incidence of neurological and other complications.

The screening for studies and retrieval of full-text articles and review was done by a single author (M.Y.Y.). Standardized Excel sheets were prepared for data extraction. Data extraction was also done by the same author (M.Y.Y.) that was cross-checked through discussion by another author (M.Q.). A risk of bias table was prepared that included information on study authors, population, location, dose of intervention, randomization generation, allocation concealment, lost to follow-up (selection bias), and blinding.

If more than one study was found with an identical outcome, then meta-analysis was conducted. For dichotomous outcomes, the effect estimate was reported as relative risks/risk ratios (RRs) and for continuous outcomes as weighted mean differences (WMDs). The a priori model of choice was fixed effects, but if substantial heterogeneity was found $\left(I^{2}>50 \%\right)$, then the random-effects model was also reported. Fixed-effects models assume that all studies estimate a single true effect size and that the variance between effect sizes is attributable to sampling error only. Random effects models assume that the studies estimate their own true effect sizes, distributed around an average true effect, where variance is attributable to both sampling error and "real" between-study variance. Random effects, therefore, give more balanced weighting to smaller studies. If heterogeneity was substantial, we used the random effects model even though we did not attempt subgroup analyses or metaregression because of the insufficient number of studies in each meta-analysis conducted.

A formal protocol of this review has not been published in any database. There was no funding source for this project.

\section{RESULTS}

A total of 107 titles/abstracts were screened, of which 57 articles were selected for detailed abstract/full-text review. One article was added from the references of the included articles. Of these 58, 15 articles or 11 distinct studies/trials [8-22] were finally selected for inclusion in this systematic review. The flow diagram of the literature search along with the reasons for 43 excluded studies is shown in Fig. 1. The risk of bias table for the 11 included studies is shown in Table 1.

\subsection{Vitamin A Supplementation for Prevention of Malaria during Pregnancy}

A total of four RCTs were identified [8,9,11-13]. Three RCTs reported on peripheral parasitemia [9,11-13], two RCTs on placental histopathological infection [8,13], one on placental infection through polymerase chain reaction (PCR) [8], and one on clinical malaria $[9,11]$. Meta-analysis could not be carried out on the outcome of peripheral parasitemia because only one study $[9,11]$ reported on incident parasitemia, i.e., new episodes after baseline assessment. In the other two studies [12,13], we could not ascertain the new cases of parasitemia at the end of pregnancy/post-partum/ follow-up, relative to baseline.

The meta-analysis based on two trials of histopathologically diagnosed placental malaria did not show evidence of benefit of vitamin A $[R R=1.09,95 \%$ confidence interval $(\mathrm{CI}), 0.95-1.25$; fixed effects, $I^{2}=0$ ] (Fig. 2). The details of these two trials are presented as follows. A randomized, double-blind placebo-controlled trial [8] was conducted in Dar es Salaam, Tanzania, where 2500 screened pregnant women during early gestation were assigned to four groups: vitamin A $2500 \mathrm{IU}$, zinc $25 \mathrm{mg}$, vitamin A $2500 \mathrm{IU}$ plus zinc $25 \mathrm{mg}$, and placebo. The treatment was given orally each day until delivery. Placentas were obtained after delivery for histopathological examination and PCR testing. In marginal structural models, vitamin A administration had no impact on histopathology-positive infection (57/679, 8\% vs. 58/682, 9\%; RR = 1.03; 95\% CI, 0.82-1.29) nor on PCR-positive placental malaria $(88 / 579,15 \%$ vs. $83 / 579,14 \%$; RR = 1.00; $95 \% \mathrm{CI}$, $0.70-1.45)$. These indicated any infection-acute, chronic, or past. All infections detected by these two methods were Plasmodium falciparum-positive. In another randomized, double-blind placebocontrolled trial [13], 98 Ghanaian women were randomized to receive 10,000 IU of vitamin A weekly or placebo. Placentas were available from 76 women and malarial infection was determined by placental blood smear and histology. All grades of placental malarial infection were 35/38 (92\%) in vitamin A group vs. $31 / 38$ (81\%) in 


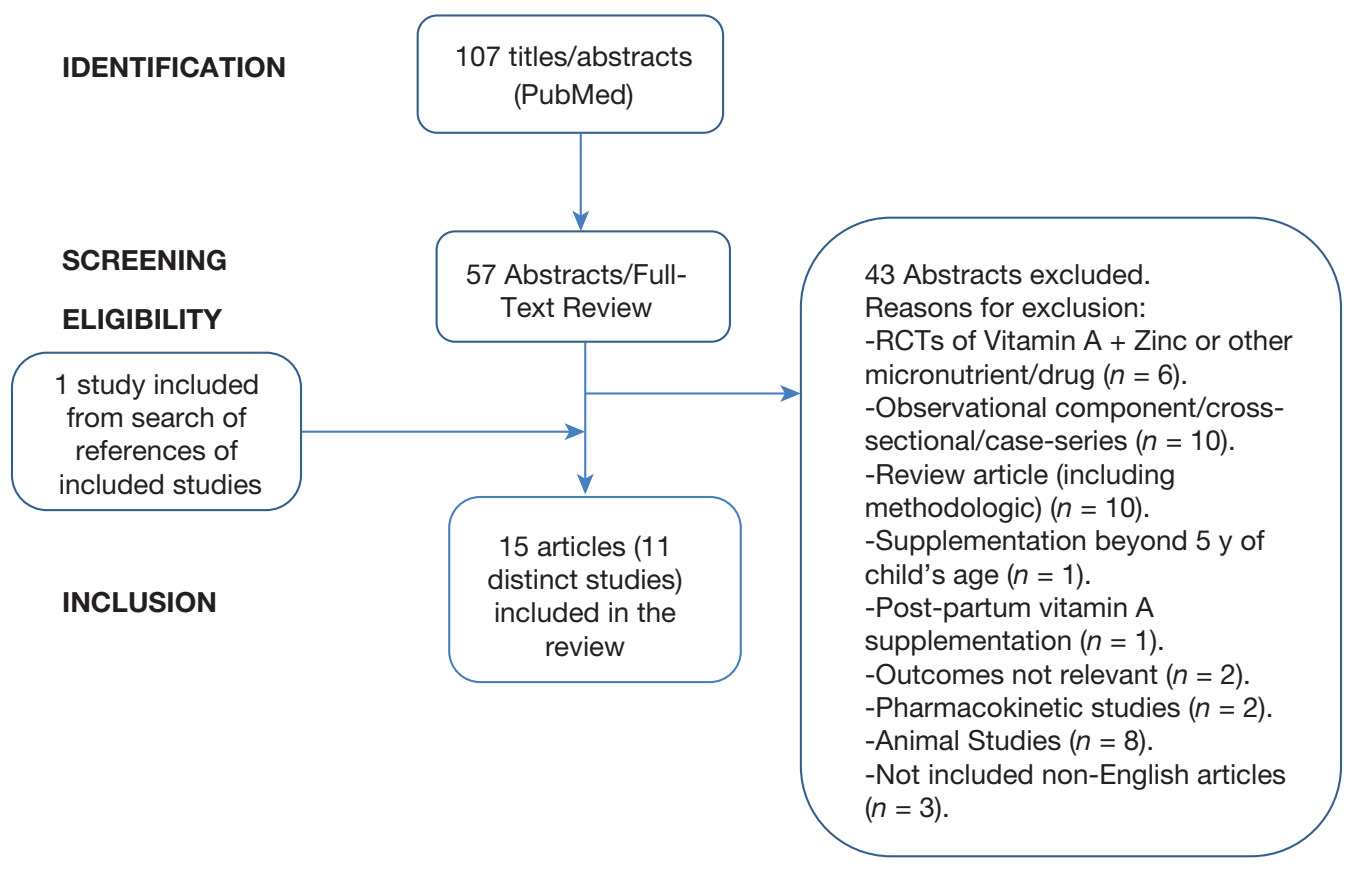

Figure 1 Flow diagram showing the steps in the literature search. RCTs, randomized controlled trials

the placebo group. Of this, active infection was $2 / 38$ (5\%) vs. $4 / 38$ (11\%), active chronic infection $12 / 38(36 \%)$ vs. $15 / 38(39 \%)$, and past-chronic infection $21 / 38(55 \%)$ vs. $12 / 38$ (32\%).

The remaining two trials were not included in any meta-analysis. In an RCT $[9,11]$ from Dar es Salaam with a factorial design, $1078 \mathrm{HIV}$-infected pregnant women were allocated into four groups to receive vitamin A alone ( $30 \mathrm{mg} \beta$-carotene with $5000 \mathrm{IU}$ preformed vitamin A), multivitamins without vitamin A, multivitamins with vitamin $\mathrm{A}$, and placebo. All women received $5 \mathrm{mg}$ folic acid and $120 \mathrm{mg}$ ferrous iron along with weekly $500 \mathrm{mg}$ chloroquine prophylaxis. The vitamin A arm was prematurely stopped because of increased risk of mother-to-child transmission of HIV. However, based on person-time contributed by this arm until second pregnancy, lost to follow-up, death, or end of study, vitamin A did not impact on the risk of developing maternal clinical malaria (rate ratio $=0.97 ; 95 \% \mathrm{CI}, 0.82-1.14 ; p=0.70$ ) nor of malarial parasitemia $(\mathrm{RR}=1.02 ; 95 \% \mathrm{CI}, 0.84-1.24 ; p=0.84)$. In a single-center RCT conducted in southern Malawi, central Africa, 700 women with singleton pregnancies with anemia were randomized to receive until delivery oral daily supplementation of 5000 or 10,000 IU vitamin A or placebo [12]. All women received iron tablets $(60 \mathrm{mg})$ and $0.25 \mathrm{mg}$ folic acid and antimalarial prophylaxis (500 mg sulfadoxine with $25 \mathrm{mg}$ pyrimethamine) at 20 and 34 weeks of gestation. There was no difference in the proportion of peripheral malaria parasitemia in the three groups at final assessment of 36-38 weeks of gestation: $13.0 \%, 17.5 \%$, and $15.0 \%(p=$ $0.56)$; similarly for parasite density $>1000(p=0.72)$.

\subsection{Vitamin A Supplementation for Treatment of Malaria during Pregnancy}

No trials were identified for vitamin A supplementation for treatment of malaria during pregnancy.

\subsection{Vitamin A Supplementation for Prevention of Malaria in Children}

A total of four RCTs were identified [14,16-19,21,22]. The details of these RCTs are mentioned below. In a double-blind randomized trial $[14,19,21,22]$, a total of 687 children between 6 and 60 months with pneumonia in Dar es Salaam, Tanzania, were randomized to receive 200,000 IU of vitamin A or placebo on the day of admission, a second dose the next day, and then at 4 and 8 months after discharge. Children younger than 12 months received 100,000 IU. Vitamin A supplementation showed a trend in reduction of malaria-specific mortality $(1 / 326,0.3 \%$ vs. $7 / 322,2.3 \%$; hazard ratio $=0.14 ; 95 \% \mathrm{CI}, 0.00-1.07 ; p=0.06)$. In Ghana, two companion high-dose vitamin A supplementation trials were conducted $[17,18]$. In the mortality Child Survival Study, 21,906 children aged 6-90 months were visited every 4 months for more than 2 years, and in the morbidity Child Health Study, 1455 children aged from 6 months to 5 years were visited weekly for 1 year. There was no difference between vitamin A supplemented versus placebo groups in malaria mortality rates (rate ratio $=1.03 ; 95 \% \mathrm{CI}, 0.74-1.43$ ) or fever incidence (nonspecific fever: rate ratio $=1.01 ; 95 \% \mathrm{CI}$, 0.96-1.07; or fever accompanied by malaise, headache, diarrhea, or vomiting: rate ratio $=1.04 ; 95 \% \mathrm{CI}, 0.92-1.18$ ). In both the trials, there were also no effects of vitamin A on malaria parasitemia rates, parasite densities in children with a positive blood smear, and rates of probable malaria illness. Based on a meta-analysis of two RCTs $[14,17]$, there was no benefit in malaria-specific mortality (rate ratio $=0.98 ; 95 \%$ CI, 0.71-1.36; fixed effects). Significant heterogeneity was found in this analysis $\left(I^{2}=72 \%\right)$, but the random effects was also nonsignificant $(\mathrm{RR}=0.49 ; 95 \% \mathrm{CI}, 0.07-3.26)$ even though the point estimate suggested $51 \%$ benefit (Fig. 3 ).

In a randomized, double-blind placebo-controlled trial, 480 children between 6 and 60 months of age were randomized to receive high dose vitamin A (200,000 IU; 100,000 IU for 


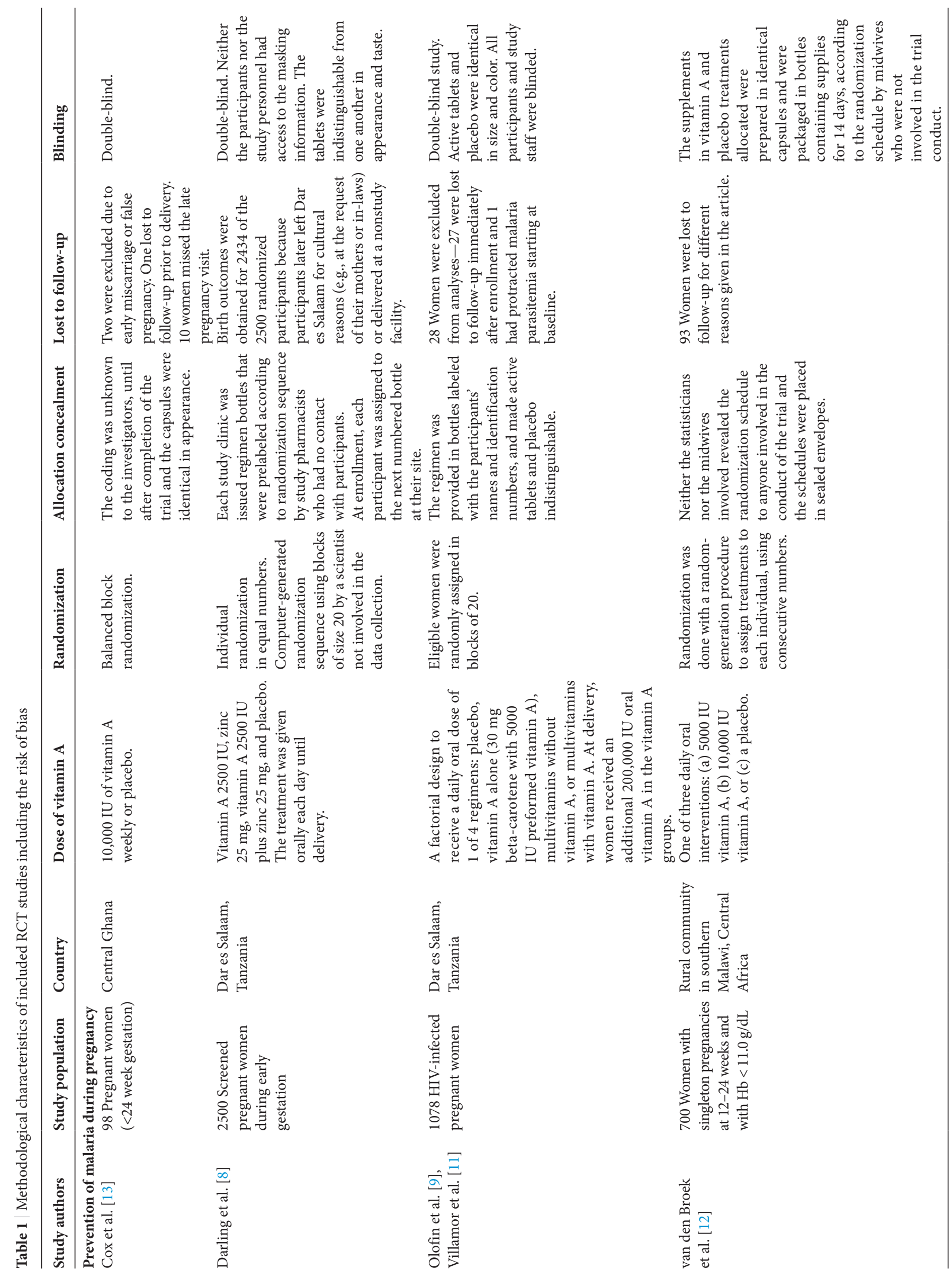




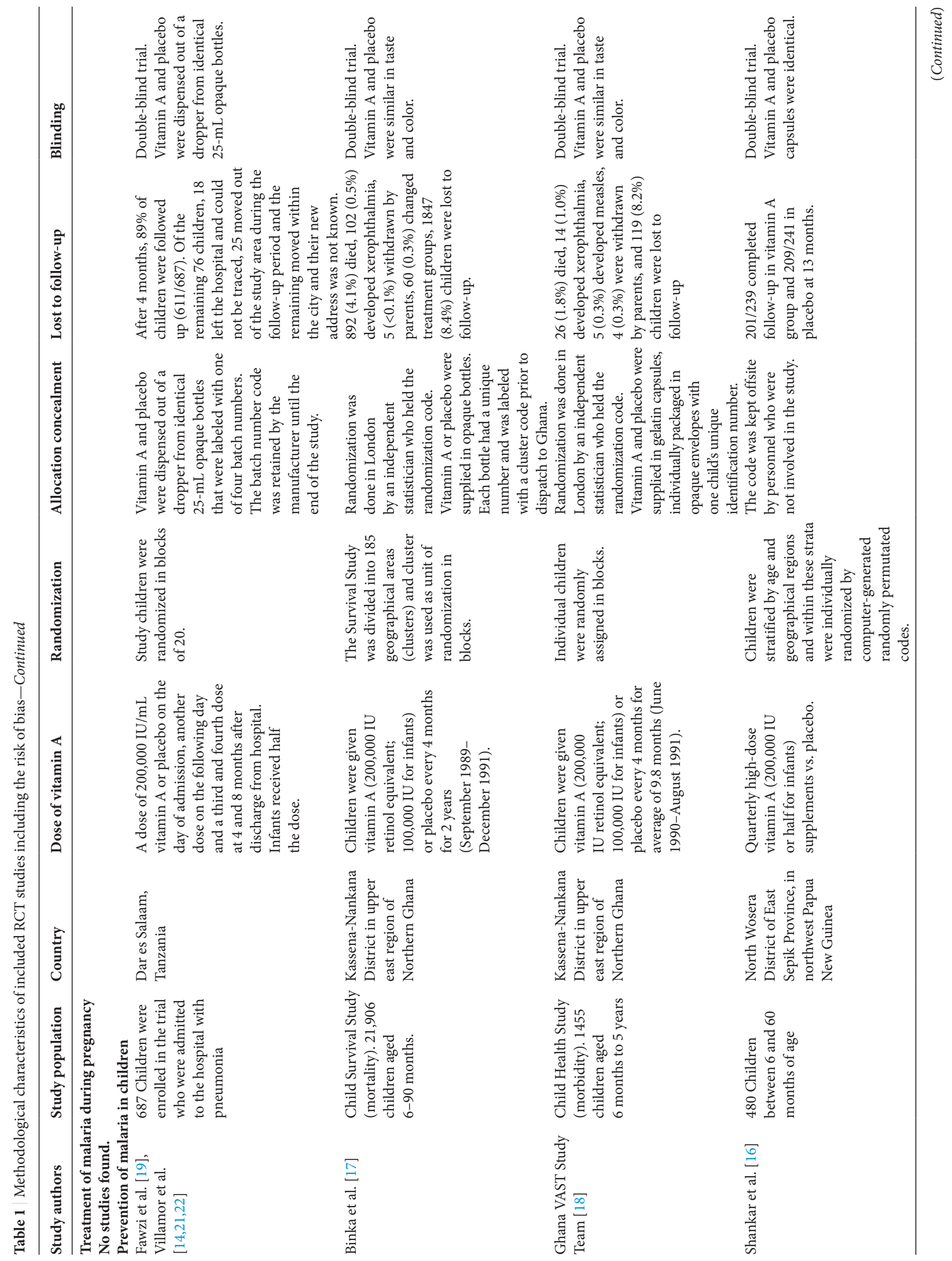




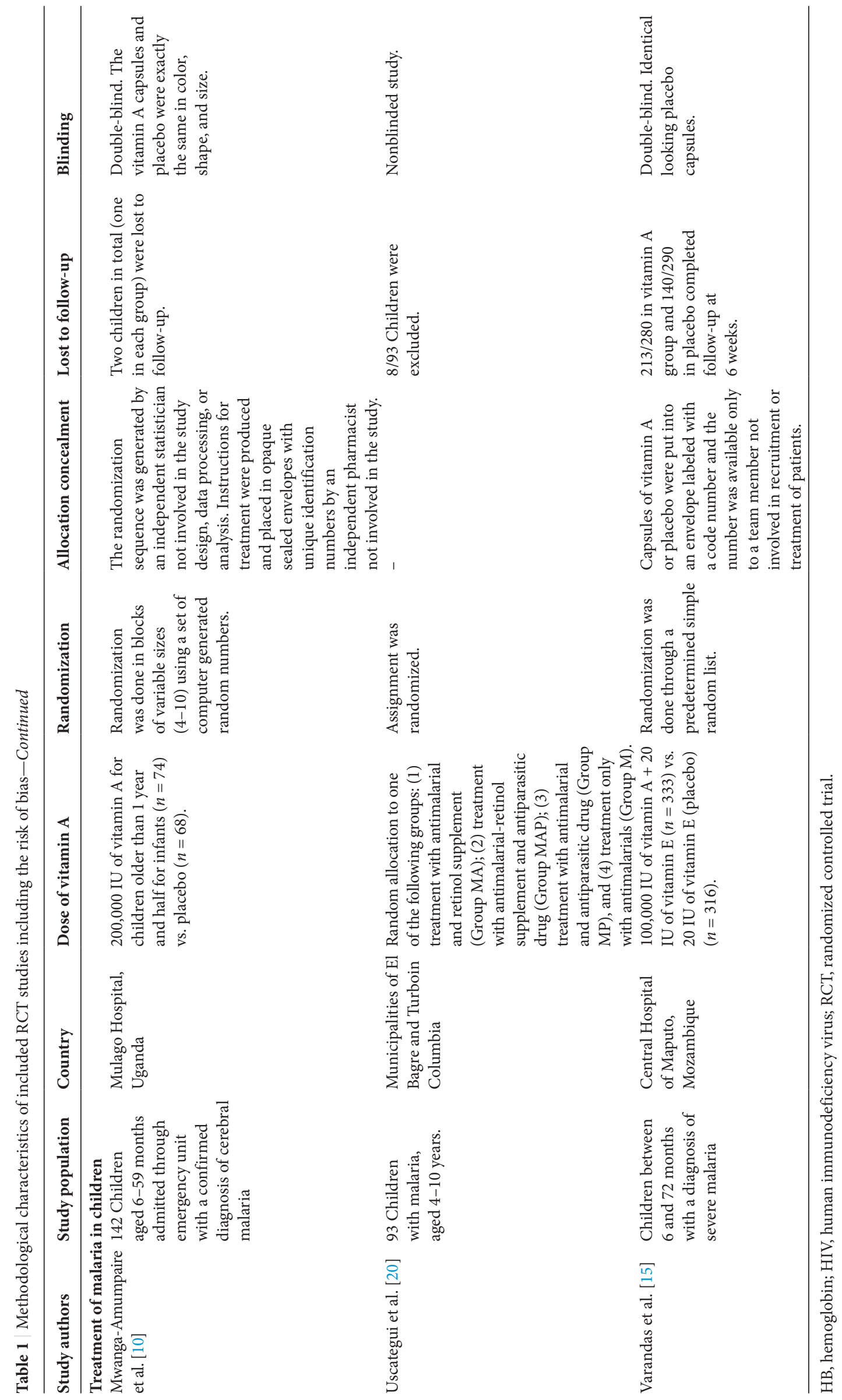




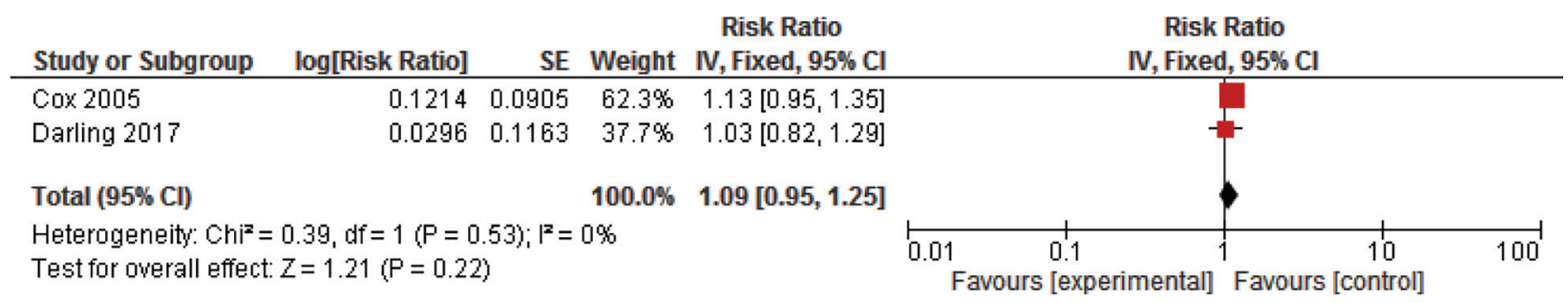

Figure 2 Meta-analysis based on two trials of the preventive effect of vitamin A supplementation on histopathologically diagnosed placental malaria infection. CI, confidence interval; SE, standard error

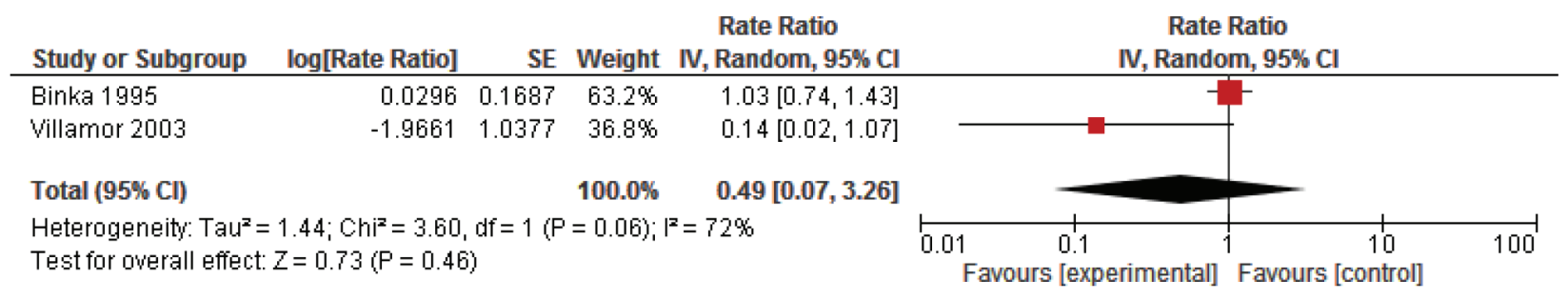

Figure 3 Meta-analysis based on two trials of the preventive effect of vitamin A supplementation on malaria-specific mortality in children younger than 5 years. CI, confidence interval; SE, standard error

$<12$ months of age) every 3 months for 13 months $(n=239)$ or placebo $(n=241)$ in Papua New Guinea [16]. There was no difference seen between groups in prevalence of parasitemia (P. falciparum, Plasmodium vivax, or Plasmodium malariae) at the end of study. There was, however, a trend toward a 36\% decrease in $P$. falciparum density (95\% CI, -8 to $62 ; p=0.093$ ) and an $11 \%(-1$ to $22 ; p=0.075)$ decrease in spleen enlargement compared with children given placebo.

\subsection{Vitamin A Supplementation for Treatment of Malaria in Children}

Three RCTs were identified [10,15,20]. One RCT had participants with cerebral malaria [10], one with severe malaria [15], and one with malaria [20]. Two RCTs reported on survival, fever and parasite clearance times, and incidence of neurological complications $[10,15]$. One RCT [20] with malaria patients as participants reported only on reduction in inflammation and other clinical outcomes not relevant to this study.

In a randomized, double-blind placebo-controlled trial [10], 142 children aged 6-59 months admitted with cerebral malaria in Kampala, Uganda were randomized to receive either vitamin A $(n=74)$ or placebo $(n=68)$ as adjunct treatment therapy. Vitamin A was administered as a single dose of 100,000 IU for those younger than 1 year and 200,000 IU for those older than 1 year. There was no difference in fever or parasite clearance times $(p=0.92$ and 0.12 , respectively) or in survival $(p=0.14)$, or occurrence of neurological complications $(p=0.44$ for coma resolution time and $p=0.37$ for resolution of convulsions) after 7 days of follow-up. In Mozambique, children aged between 6 and 72 months admitted with diagnosis of severe malaria were randomized to a single oral dose of 200,000 IU (100,000 IU if $<12$ months old) vitamin A $(n=280)$ or placebo $(n=290)$ and followed up to 6 weeks after discharge [15]. Seven of 277 (2.5\%) and 13 of 288 (4.5\%) children died in the experimental and control groups ( $\mathrm{RR}$ of death $=0.56 ; 95 \%$ CI, $0.23-1.38 ; p=0.201$ ). This difference was clinically rather than statistically important. The risk of death, however, was significantly higher in vitamin A group compared with placebo 5 hours after admission but not during the first 5 hours. Overall, 4/82 (4.9\%) vs. $2 / 78$ (2.6\%) among patients with cerebral malaria developed neurological sequelae $(\mathrm{RR}=1.90 ; 95 \% \mathrm{CI}, 0.36-10.09 ; p=0.682)$. The incidence of frequency of complications $(\mathrm{RR}=0.92 ; 95 \% \mathrm{CI}, 0.66$ $1.29 ; p=0.623)$ and blood transfusion $(\mathrm{RR}=0.94 ; 95 \% \mathrm{CI}, 0.87-$ $1.02 ; p=0.161$ ) was not different between vitamin A supplemented and placebo groups. The time to resolution of fever $(p=0.151)$, resolution of coma $(96 / 102$ vs. $88 / 99 ; p=0.746)$, and clearance of parasitemia were not different $(73 / 123$ vs. $88 / 164 ; p=0.833)$.

A nonblinded, randomized experimental study [20] was conducted in 93 children with malaria aged 4-10 years. The children were allocated into four groups: (1) treatment with antimalarial and retinol supplement, (2) treatment with antimalarial-retinol supplement and antiparasitic drug, (3) treatment with antimalarial and antiparasitic drug, and (4) treatment only with antimalarials. The groups were observed for 30 days. Inflammation was measured in terms of C-reactive protein $>8 \mathrm{mg} / \mathrm{L}$. Although in total, inflammation was reduced from $96 \%$ to $18 \%$, there was no difference in frequency of inflammation either at baseline or end of study among the groups ( $p=0.512$ and 0.454 , respectively). This indicates that inflammation was reduced in all groups, which could be attributable to antimalarials, and vitamin A did not have any specific effect in reducing inflammation related to malarial infection.

\section{DISCUSSION}

In this review, we found that vitamin A supplementation during pregnancy had no benefit in reducing placental malarial infection nor in peripheral parasitemia. Similarly, vitamin A supplementation in children had no impact on malaria-specific mortality. 
Although prior evidence has shown that vitamin A reduces diarrhea and measles incidence, this review shows that it has no such protective effect either for malaria incidence or for malaria-specific mortality. Regarding treatment effects of vitamin A as an adjunct therapy, based on RCTs of children with cerebral or severe malaria, there was no difference between the groups in survival, fever and parasite clearance times, or in incidence of neurological sequelae or other complications. Similarly, no benefit was found for prevention of malaria. According to the best of our knowledge, this is the first systematic review and meta-analysis studying vitamin A supplementation for both prevention and treatment of malaria.

Different animal model and in vitro studies have been conducted to study the role of vitamin A supplementation in decreasing malarial parasitemia or infection intensity with conflicting results. An animal study [23] on mice showed that vitamin A supplementation alone had no effect on parasitemia development and even increased the risk when administered with diphtheria-tetanuspertussis (DTP) vaccine. The authors had earlier concluded [24] that it depended on the pathogenesis of disease because vitamin A plus DTP tended to increase parasitemia and significantly suppressed cytokine responses in mice with cerebral malaria, but this was not the case in mice dying of anemia. Similarly, another murine model study [25] showed that retinol, when administered prior to Plasmodium berghei inoculation, reduced end-point parasitemia by $24 \%$ ( $p=0.001$ vs. controls) but was not effective when given both prior to and after infection ( $11 \%$ reduction, $p=0.47)$. This effect of retinol was mediated through increase in tissue and liver retinol stores rather than to changes in oxidant status. In an in vitro study of $P$. falciparum [26], all stages of parasite development including merozoite invasion were sensitive to retinol levels. There was evidence of membrane rupture of parasites and increased vacuolization of parasite food particles with retinol treatment. This indicated a potential antimalarial role of vitamin $\mathrm{A}$ as these effects were similar to those seen with quinoline and artemisinin compounds. In another animal study in rats with $P$. berghei infection [27], parasitemia developed at a faster pace in vitamin A, protein energyundernourished rats compared with those supplemented with vitamin A. A higher number of red blood cells infected with the parasite was noticeable 6-7 days after the infection, at which point most of the animals in the former group died.

In contrast to mixed findings from animal studies, human cross-sectional and observational studies have more consistently indicated an inverse association between vitamin A supplementation and malarial parasitemia. In a cross-sectional study based on 39 individuals, there was an inverse correlation of retinol levels with parasitemia $(r=-0.338, p=0.035)$ [28]. In a recent observational national survey from sub-Saharan Africa [29], in more than 8000 children aged 6-59 months over an 18-month period, vitamin A supplements reduced parasitemia by $54 \%(\mathrm{RR}=0.46 ; 95 \%$ CI, 0.39-0.54) and P. falciparum-related antigenemia by $77 \%$ (RR $=0.23 ; 95 \% \mathrm{CI}, 0.17-0.29)$. In a secondary analysis of a clusterrandomized trial of peer counseling for exclusive breastfeeding [30], among 483 infants aged between 3 and 12 months who did not receive vitamin A, there was a higher risk of prevalent parasitemia compared with those who received vitamin A after controlling for use of bed nets, age, and residence (prevalence ratio $=6.1 ; 95 \%$ CI, 2.1-17.6). Because of the study design and limited adjustment for confounding, the cause-effect relationship in these studies, however, remains unclear. Furthermore, our meta-analysis based on RCT evidence does not show a benefit or support the findings of these studies.

The main strengths of our review include a comprehensive, systematic search to identify RCTs on the topic, compiling evidence on both preventive and treatment roles of vitamin A in pregnancy and childhood, and conducting meta-analyses where more than one outcome was found-for placental malarial infection (for supplementation in pregnancy) and malaria-specific mortality (for supplementation in childhood). The main limitation was inability to distinguish prevalent versus incident peripheral parasitemia from published articles that precluded meta-analysis of this outcome.

\section{CONCLUSION}

We did not find any evidence of benefit of vitamin A supplementation during pregnancy on peripheral parasitemia or placental malarial infection. Similarly, the effects of supplementation in children also showed no benefit either in parasitemia or mortality rates due to malaria. Potential reasons should be explored as to why cross-sectional and observational studies have shown a benefit, whereas RCT evidence shows otherwise; moreover, differences in findings should be reconciled through more studies if needed.

\section{CONFLICTS OF INTEREST}

The authors declare that they have no conflicts of interest.

\section{REFERENCES}

[1] Global Burden of Disease 2016. 2016. Available from: https:// vizhub.healthdata.org/gbd-compare/ (accessed October 10, 2016).

[2] World Health Organization. Fact Sheet: World Malaria Report 2016. Available from: http://www.who.int/malaria/media/ world-malaria-report-2016/en/ (accessed October 23, 2016).

[3] Caulfield LE, Richard SA, Black RE. Undernutrition as an underlying cause of malaria morbidity and mortality in children less than five years old. Am J Trop Med Hyg 2004;71;55-63.

[4] Shankar AH. Nutritional modulation of malaria morbidity and mortality. J Infect Dis 2000;182;S37-53.

[5] Imdad A, Mayo-Wilson E, Herzer K, Bhutta ZA. Vitamin A supplementation for preventing morbidity and mortality in children from six months to five years of age. Cochrane Database Syst Rev 2017;3;CD008524.

[6] McCauley ME, van den Broek N, Dou L, Othman M. Vitamin A supplementation during pregnancy for maternal and newborn outcomes. Cochrane Database Syst Rev 2015;10;CD008666.

[7] Semba RD. Vitamin A and immunity to viral, bacterial and protozoan infections. Proc Nutr Soc 1999;58;719-27.

[8] Darling AM, Mugusi FM, Etheredge AJ, Gunaratna NS, Abioye AI, Aboud S, et al. Vitamin A and zinc supplementation among pregnant women to prevent placental malaria: a randomized, double-blind, placebo-controlled trial in Tanzania. Am J Trop Med Hyg 2017;96;826-34.

[9] Olofin IO, Spiegelman D, Aboud S, Duggan C, Danaei G, Fawzi WW. Supplementation with multivitamins and vitamin A and incidence of malaria among HIV-infected Tanzanian women. J Acquir Immune Defic Syndr 2014;67;S173-S78. 
[10] Mwanga-Amumpaire J, Ndeezi G, Tumwine JK. Effect of vitamin A adjunct therapy for cerebral malaria in children admitted to Mulago hospital: a randomized controlled trial. Afr Health Sci 2012;12;90-7.

[11] Villamor E, Msamanga G, Saathoff E, Fataki M, Manji K, Fawzi WW. Effects of maternal vitamin supplements on malaria in children born to HIV-infected women. Am J Trop Med Hyg 2007;76;1066-71.

[12] van den Broek NR, White SA, Flowers C, Cook JD, Letsky EA, Tanumihardjo SA, et al. Randomised trial of vitamin A supplementation in pregnant women in rural Malawi found to be anaemic on screening by HemoCue. BJOG 2006;113;569-76.

[13] Cox SE, Staalsoe T, Arthur P, Bulmer JN, Tagbor H, Hviid L, et al. Maternal vitamin A supplementation and immunity to malaria in pregnancy in Ghanaian primigravids. Trop Med Int Health 2005;10;1286-97.

[14] Villamor E, Fataki MR, Mbise RL, Fawzi WW. Malaria parasitaemia in relation to HIV status and vitamin A supplementation among pre-school children. Trop Med Int Health 2003;8; 1051-61.

[15] Varandas L, Julien M, Gomes A, Rodrigues P, Van Lerberghe W, Malveiro F, et al. A randomised, double-blind, placebocontrolled clinical trial of vitamin A in severe malaria in hospitalised Mozambican children. Ann Trop Paediatr 2001;21;211-22.

[16] Shankar AH, Genton B, Semba RD, Baisor M, Paino J, Tamja S, et al. Effect of vitamin A supplementation on morbidity due to Plasmodium falciparum in young children in Papua New Guinea: a randomised trial. Lancet 1999;354;203-9.

[17] Binka FN, Ross DA, Morris SS, Kirkwood BR, Arthur P, Dollimore N, et al. Vitamin A supplementation and childhood malaria in northern Ghana. Am J Clin Nutr 1995;61;853-59.

[18] Vitamin A supplementation in northern Ghana: effects on clinic attendances, hospital admissions, and child mortality. Ghana VAST Study Team. Lancet 1993;342;7-12.

[19] Fawzi WW, Mbise RL, Hertzmark E, Fataki MR, Herrera MG, Ndossi G, et al. A randomized trial of vitamin A supplements in relation to mortality among human immunodeficiency virusinfected and uninfected children in Tanzania. Pediatr Infect Dis J $1999 ; 18 ; 127-33$.

[20] Uscátegui RM, Correa AM, Carmona-Fonseca J. [Changes in retinol, hemoglobin and ferritin concentrations in Colombian children with malaria]. Biomedica 2009;29;270-81 (in Spanish).
[21] Villamor E, Mbise R, Spiegelman D, Ndossi G, Fawzi WW. Vitamin A supplementation and other predictors of anemia among children from Dar es Salaam, Tanzania. Am J Trop Med Hyg 2000;62; 590-97.

[22] Villamor E, Mbise R, Spiegelman D, Hertzmark E, Fataki M, Peterson KE, et al. Vitamin A supplements ameliorate the adverse effect of HIV-1, malaria, and diarrheal infections on child growth. Pediatrics 2002;109; e6.

[23] Jørgensen MJ, Hein-Kristensen L, Hempel C, Ravn H, Wiese L, Kurtzhals JA, et al. The effect of vitamin A supplementation and diphtheria-tetanus-pertussis vaccination on parasitaemia in an experimental murine malaria model. Scand J Infect Dis 2011;43;296-303.

[24] Hein-Kristensen L, Jørgensen MJ, Ravn H, Wiese L, Kurtzhals J, Benn CS. Simultaneous administration of vitamin A and DTP vaccine modulates the immune response in a murine cerebral malaria model. Scand J Immunol 2010;72;302-8.

[25] Hamzah J, Batty KT, Davis WA, Mori TA, Ching SY, Croft KD, et al. Retinol supplementation in murine Plasmodium berghei malaria: effects on tissue levels, parasitaemia and lipid peroxidation. Int J Parasitol 2007;37;525-37.

[26] Hamzah J, Davis TM, Skinner-Adams TS, Beilby J. Characterization of the effect of retinol on Plasmodium falciparum in vitro. Exp Parasitol 2004;107;136-44.

[27] Krishnan S, Krishnan AD, Mustafa AS, Talwar GP, Ramalingaswami V. Effect of vitamin A and undernutrition on the susceptibility of rodents to a malarial parasite Plasmodium berghei. J Nutr 1976;106;784-91.

[28] Tabone MD, Muanza K, Lyagoubi M, Jardel C, Pied S, AmedeeManesme $\mathrm{O}$, et al. The role of interleukin-6 in vitamin A deficiency during Plasmodium falciparum malaria and possible consequences for vitamin A supplementation. Immunology 1992;75;553-54.

[29] Hollm-Delgado MG, Piel FB, Weiss DJ, Howes RE, Stuart EA, Hay SI, et al. Vitamin A supplements, routine immunization, and the subsequent risk of Plasmodium infection among children under 5 years in sub-Saharan Africa. Elife 2015;4;e03925.

[30] Nankabirwa V, Tylleskar T, Nankunda J, Engebretsen IM, Sommerfelt H, Tumwine JK. Malaria parasitaemia among infants and its association with breastfeeding peer counselling and vitamin A supplementation: a secondary analysis of a cluster randomized trial. PLoS One 2011;6;e21862. 